\title{
XVII.
}

\section{Ueber Muskel-Paralyse, als Ursache der Gelenkverkrümmungen.}

Ein Beitrag zur Aetiologie und Therapie der Deformitäten.

Von Dr. Eulenburg,

pract. Arzte, Operateur und Geburtshelfer, Director des Instituts für schwedische

Heilgymnastik und Orthopädie zu Berlin.

Die Therapie der Deformitäten mufs sich, wie die rationelle Behandlung einer jeden anderen Krankheit, mit möglichster Consequenz auf die primären ursächlichen Momente stützen. Ohne Würdigung der pathologischen Verhältnisse entbehrt jede Behandlung des wissenschaftlichen Haltes und verfällt der crassen Empirie. Die Behandlung der Deformitäten konnte nur dadurch den Händen der Bandagisten und Mechaniker vèrfallen, dafs man dieselbe vom rein mechanischen Gesichtspunkte aus auffafste, ihren organischen Ursprung und Zusammenhang aber gänzlich übersah.

Seitdem die gediegensten Aerzte und Chirurgen den vitalen Ursachen der Deformitäten eine gröfsere Beachtung gewidmet haben, seitdem die Heroen der Physiologie und pathologischen Anatomie auch dies Gebiet gründlicher zu erforschen begannen, stand zu erwarten, dals auch dessen Therapie aus der geläuterten physiologischen Auffassung ihren Gewinn ziehen würde. Allein Jahrhunderte alte Vorurtheile werden nicht in kurzer Zeit beseitigt, sie müssen als solche immer wieder bekämpft werden, wenn sie einer richtigen Erkenntnifs weichen sollen. Daher kann 
die wiederholte Erörterung des Gegenstandes nicht überflüssig sein. Aehnliches ward vielleicht von Anderen schon gesagt, dennoch hoffe ich, dafs mancher Leser einige neue Gesichtspunkte von praktischem Werthe aus meiner Darstellung gewinnen werde. Ich selbst wenigstens studire mit nicht geringem Intewesse die Arbeiten anderer Autoren, wenn ich in denselben auch nur die Bestätigung von Ansichten wiederfinde, deren Wahrheit bei mir längst zur Ueberzeugung geworden war.

-So verweile ich mit Vergnügen bei den neuerdings erschienenen Vorträgen B ouvier's (Ueber die chronischen Krankheiten des Bewegungs-Apparates der Kinder. Journal für Kinderkrankheiten 1855. Hft. 9 u. 10. S.262. u. 1856. Hft. 1. u. 2. S.89-113.), Vorlesungen, von welchen man sagen kann, dafs Form und Inhalt unübertrefflich sind. B ouvier giebt hier in der Einleitung mit wenigen Worten eine Uebersicht der Entstehung chronischer Krankheiten des Bewegungsapparates. Er weist nach, wie die 3 Systeme desselben: das Muskelsystem, das die Muskeln versorgende Nervensystem und das Knochensystem mit seinen Knorpeln, Bändern und Synovialmembranen eine Art hierarchischer Trilogie bilden, sich einander unterordnen, sowohl in physiologischer als in pathologischer Hinsicht. Wie im Normalacte der Locomotion, ebenso greifen diese 3 Systeme auch in den krankhaften Aeufserungen derselben ineinander und eines folgt dem anderen botmäfsig. Der Nerv erregt den Muskel, der Muskel wirkt auf den Knochen, der Knochen wird in Bewegung gesetzt. Es giebt demnach, so folgert Bouvier, eine Reihe. Störungen des Knochensystems, die von Erkrankungen der Muskeln herrühren und es giebt eine Reihe Störungen des Knochen- und Muskelsystems, deren Quelle das Nervensystem ist. - Aufserdem können das Knochensystem unabhängig vom Muskel- und Nervensystem und ebenso die Muskeln unabhängig von dem über sie gebietenden Nervensysteme erkranken. Wende ich diese vorangestellte Anschauung der Krankheiten des Bewegungsapparates auf die Deformitäten an, welche mit Gelenkverkrümmungen verbunden sind, so ergiebt sich für dieselben im Wesentlichen meine bereits früher aufgestellte ursächliche Einthei- 
lung (s. Meine Mittheilungen aus dem Gebiete der schwedischen Heilgymnastik. 'Berlin 1854 bei A. Hirschwald.). Sie können entstehen:

1) Aus primärer Erkrankung des betreffenden activen'Bewegungsapparates und zwar kann diese beruhen:

a) auf gemeinsamer Störung des Nerven- und Muskelsystems in Form von Paralyse, Subparalyse, Relaxation oder in Form von permanenter Muskelverkürzung in Folge eines Nerveneinflusses (tonische Contraclion),

b) auf alleiniger Störung des Muskelsystems in Folge von traumatischen, rheumatischen oder ähnlichen Affectionen der Muskeln;

2) aus primärer Erkrankung des das Gelenk constituirenden passiven Bewegungsapparates, d. h. der Knochen, Knorpel, Fascien, Bänder und Synovialmembranen.

Diese das Gelenk constituirenden passiven Bewegungsorgane können nun

a) einzeln oder

b) in ihrer Gesammtheit

erkranken und Veranlassung zu mannigfachen Deformitälen werden.

Brodie hat zuerst den Versuch gemacht, die Gelenkkrankheiten nach Affection der einzelnen Gewebstheile darzustellen. Auf Brodie's darüber handelndes Werk sich stïtzend, hat Dr. G. A. So er unter dem bescheidenen Namen einer Uebersetzung (Abhandlung über die Krankheiten der Gelenke von B. C. Brodie, aus dem Englischen von Dr. G. A. Soer, Coblenz 1853.) eine eigene, unendlich verdienstvolle Arbeit geliefert, in welcher er mit gesundester Kritik Brodie's reiche Erfahrung benutzte, um daran dessen Ansichten zu einem festen Systeme auszubauen.

Ich werde später auf die sub2. als aus der primären Erkrankung der passiven Bewegungsorgane hervorgegangenen Deformitäten nochmals zurïckkommen. Hier sei nur bemerkt, dafs ihre differentielle Diágnose zwar mit der der er- 
krankten Gewebe identisch ist, sie aber an Schwierigkeit noch dadurch übertrifft, dals die Deformität, wenn sie als solche in Behandlung kommt, meistens der unglückliche Ausgang eines längst abgelaufenen complicirten Krankheitsprozesses ist.

Ich wende mich nunmehr zu meiner vorgesetzlen Aufgabe, als der sub 1. angeführten Species von Deformitäten:

Dahin zähhle ich diejenigen die Gelenke in sich schliefsenden Deformitäten, welche ursprünglich das betreffende Gelenk als solches in keiner anderen Weise, als in seiner normalen Stellung alteriren. Diese Gelenkdeformitäten nenne ich im Allgemeinen: musculäre Gelenkverkrümmung en, und betrachte sie als völlig analog mit den von mir anderweitig erörterten musculären Rückgratsverkrümmungen. Am deutlichsten ausgeprägt erscheint die musculäre Störung bei den Deformitäten der unteren Extremitäten.

Die musculäre Deformität beruht entweder auf Muskelretraction oder - auf Muskelparalyse. Der ersteren hat man mit Unrecht ein bei Weitem grölseres Gebiet in der Aetiologie der Deformitäten eingeräumt, als der Paralyse, ja man hat diese Paralyse, die ich in ihren verschiedenen Abstufungen als die häufigste Ursache der Deformitäten betrachte, oft ganz übersehen und hat, wie ich in einem Nachtrage zu dieser Arbeit speciell nachweisen werde, sich bemüht, völlig secundäre $\mathrm{Zu}-$ stände als das Wesen der Deformität hinzustellen.

In meinen früheren Arbeiten über Scoliose habe ich dargethan, wie durch die verminderte Energie einzelner Muskelgruppen und die dadurch eingetretene Störung des Antagonismus, die gesunden Antagonisten gezwungen sind, sich zu verkïrzen und dadurch die Wirbelsäule concav nach ihrer Seite zu krïmmen (s. u. A. meinen Aufsatz: "Beiträge zur Lehre von den Rückgratsverkrürnmungen" im Journal fiir Kinderkrankheiten. 1856. Heft 1 u. 2.).

Ganz auf dieselbe Weise entsteht eine überaus grofse Zahl von Gelenkdeformitäten an den Extremitäten. Durch die verminderte Energie einer an einem Gelenke thätigen Muskelgruppe isl diese behindert, den Antagonisten genügenden Widerstand 
zu leisten. Diese sind dadurch gezwungen, sich um soviel zu verkürzen, als jene an Contractionskraft eingebülst hat. Die dadurch bewirkte Deformität wird um so grölser sein, jemehr sich die mangelhafte Innervation der völligen Paralyse nähert. Umgekehrt werden der Form nach zwar ähnliche, dem pathogenetischen Wesen nach aber ganz verschiedene Deformitäten entstehen, wenn einzelne an einem Gelenke thätige Muskeln oder Muskelgruppen in ihrem Gewebe krankhaft sich verkürzen (retrahiren) und durch diese Retraction ihren Antagonisten ein Hindernils setzen, normal zu agiren. In beiden Fällen sind es gleichwohl die pathischen Zustände der Nerven und Muskeln allein, durch welche die übrigen das Gelenk constituirenden passiven Bewegungsorgane: Knochen, Knorpel, Ligamente u. s.w. in die abnorme Stellung gebracht werden. Um diese an sich schon verständliche Auffassung durch eine leichte Uebersicht noch anschaulicher zu machen, benutze ich nachstehend eine von Valentin aufgestellte, von mir ergänzte Tabelle.

In Columna I. dieser befinden sich die Namen der gewöhnlichsten musculären Gelenkdeformitäten der Unterextremitäten; in Columna II. sind die Muskeln angegeben, durch deren Paralyse und in Columna III. diejenigen Muskeln, durch deren Retraction die in Columna I, angeführte Deformität entstehen kann.

Ein Vergleich der unter Columna II und III. neben einander stehenden Muskeln ergiebt, dafs diese im normalen Zustande in einem antagonistischen Zusammenhange stehen (s. Valentin's Lehrbuch der Physiologie 1847. Bd. II. Abthl. I. S. 329.)

\begin{tabular}{|c|c|c|}
\hline $\begin{array}{c}\text { I. } \\
\text { Deformität. }\end{array}$ & $\begin{array}{l}\text { II. } \\
\text { Paralysirte Muskeln. }\end{array}$ & $\begin{array}{l}\text { III. } \\
\text { Retrahirte Muskeln. }\end{array}$ \\
\hline $\begin{array}{l}\text { 1) Beugung d. Oberschen- } \\
\text { kels im Hüftgelenke. }\end{array}$ & $\begin{array}{l}\text { Mm. glutaei, bes. Glutaeus } \\
\text { maximus, Obturator in- } \\
\text { termus; Caput longum bi- } \\
\text { cipitis; semitendinosus } \\
\text { semimembranosus; gra- } \\
\text { cilis. }\end{array}$ & $\begin{array}{l}\text { M. iliopsoas oder Rectus fe- } \\
\text { moris; sartorius; pectineus. }\end{array}$ \\
\hline $\begin{array}{l}\text { 2) Adduction des 0ber- } \\
\text { schenkels. }\end{array}$ & $\begin{array}{l}\text { Mm. glutaei; tensor fa- } \\
\text { sciae latae. }\end{array}$ & $\begin{array}{l}\text { M. pectineus; Adductor fo- } \\
\text { moris longus, brevis und } \\
\text { magnus. }\end{array}$ \\
\hline $\begin{array}{l}\text { 3) Beugung im Knie (des } \\
\text { Unterschẹnkels). }\end{array}$ & $\begin{array}{l}\text { M. rectus femoris; crura- } \\
\text { lis; vasti ex- } 11 \text { interni; } \\
\text { sartorius. }\end{array}$ & $\begin{array}{l}\text { M. biceps femoris; semitendi- } \\
\text { nosus, semimembranosus, } \\
\text { popliteus. }\end{array}$ \\
\hline
\end{tabular}




\begin{tabular}{l} 
Deformität. \\
\hline 4) Abnorme Streckung im \\
Knie (des Untersehen- \\
kels) wird durch die der \\
Beugung entgegenge- \\
setzten Muskelverhält- \\
nisse bedingt. \\
5) Verbiegung des Kniege- \\
lenkes nach aussen (Sä- \\
belbein, Genu varum). \\
6) Verbiegung des Kniege- \\
lenkes n. innen (Sche- \\
melbein, Genuvalgum). \\
7) Einfacher Pferde- oder \\
Spitzfuss. \\
8) Unreiner Pferde- oder \\
Spitzfuss.
\end{tabular}

9) Einfacher Klumpfuss.

10) Fersen - Klumpfuss (Talipes calcaneus).

11) Pferdefuss-Klumpfuss (Talipes varo-equinus).

12) Plattfuss (Pes valgus).

13) Hohifuss.

14) Einwärtswendung des Fusses.
II. Paralysirte Muskeln.

III. Retrahirte Muskeln.

M. biceps; semilendinosus, semimembranosus, poM, rectus femoris, cruralis, pliteus (gastrocnemius).

M. biceps femoris.

M. popliteus, gracilis, semilendinosus (semimembranosus, sartorius).

M. tibialis anticus, peraneus tertius.

M. tibialis anticus, peroneus tertius, extensores digitorum pedis communes longi, extensores hallucis.

M. peronei longus, brevis und tertius.

M. peronei longus und brevis, flexores digitorum, flexor hatlucis longus.

M. peronei longus, brevis und tertius, extensor digitor. pedis communis, extensor hallucis.

M. tibialis posticus und anticus.

M. peronei brevis und tertius, adductor hallucts, extensor digitor. pedis communis longus und brevis.

M. peronei longus und M. tibialis posticus.

Analoge pathische Verhältnisse finden sich an den Oberextremitäten. Zahlreiche Beobachtungen setzen die Thatsache aufser Zweifel, dafs die Deformitäten in einer in verschiedenen Graden bestehenden, oft bis zur gänzlichen Unthätigkeit gedie- 
henen Paralyse einzelner Muskeln oder Muskelgruppen ihren primären Grund haben. Wir wissen nun aber, dafs die Thätigkeit der Muskeln abhängig ist von der normalen. Function der über sie gebietenden Nerven. Um die Unthäligkeit einzelner Muskelgruppen bei gleichzeitiger Integrität ihrer Antagonisten physiologisch zu erklären, bedarf es freilich des anatomischen Nachweises, dafs- die gelähmten Muskeln nicht von denselben Nervenästen versorgt werden, wie ihre Antagonisten. Hier begegnen -wir einer grofsen Schwierigkeit. Wir sehen zwar sehr häufig eine wesentliche Sonderung der Nerven nach der antagonistischen Function der ihnen untergeordneten Muskeln, oft aber verbreiten sich auch Nerven aus demselben Aste in Muskeln, welche zu einander in antagonistischer Beziehung stehen.

Die unteren Extremitäten, an welchen die von Paralyse abhängigen Deformitäten so überwiegend häufig sind, gestatten auch einen ziemlich vollständigen Nachweis, dafs die im antagonistischen Verhältnisse fungirenden Muskeln von getrennten Nervenästen beherrscht werden.

Folgende Zusammenstellung in antagonistischer Beziehung zu einander stehender Muskeln mag diese Ansicht erläutern.

\begin{tabular}{|c|c|c|c|}
\hline $\begin{array}{l}\text { I. } \\
\text { tagonistische Mus- } \\
\text { ln von Columna III. }\end{array}$ & $\begin{array}{c}\text { II. } \\
\text { Versorgende Nerven. }\end{array}$ & $\begin{array}{c}\text { Antagonistische Mus- } \\
\text { keln von Columna I. }\end{array}$ & $\begin{array}{l}\text { IV. } \\
\text { Versorgende } \\
\text { Nerven. }\end{array}$ \\
\hline $\begin{array}{l}\mathrm{B} \\
\mathrm{H}\end{array}$ & $\begin{array}{l}l u \\
e x\end{array}$ & skeln d. & $\begin{array}{l}N . \\
N . \\
u s .\end{array}$ \\
\hline $\begin{array}{l}\text { Die Einw } \\
\text { des Obers }\end{array}$ & $\begin{array}{l}\text { N. } g \\
\text { N. } \\
\text { dic }\end{array}$ & $\begin{array}{c}\text { Die } \\
\mathrm{Ob}\end{array}$ & $\begin{array}{l}N . \\
N . \\
N n\end{array}$ \\
\hline $\begin{array}{l}\text { Die Beu } \\
\text { Oberscher }\end{array}$ & $\begin{array}{l}\text { Vord } \\
\text { lum } \\
\text { ext }\end{array}$ & $\begin{array}{r}\text { Dies } \\
\text { Ob }\end{array}$ & $\begin{array}{l}\text { Nn. g } \\
\text { plexu } \\
\text { dicus. }\end{array}$ \\
\hline $\begin{array}{l}\text { Die Anzi } \\
\text { Obersche }\end{array}$ & $\begin{array}{r}\text { N. ob } \\
\text { dicu }\end{array}$ & $\begin{array}{c}\mathrm{Die} \\
\mathrm{scl}\end{array}$ & $\begin{array}{l}\text { N. glutaei, } \boldsymbol{N} \\
\text { cruralis. }\end{array}$ \\
\hline 5) $\underset{\text { ter }}{\mathrm{Di}}$ & $\begin{array}{l}\text { iadicus, } N . p e- \\
\text { es. }\end{array}$ & $\begin{array}{r}\text { Die } \\
\text { sch }\end{array}$ & N. $c r u$ \\
\hline 6) Ein & $\begin{array}{l}\text { cus u. } N \text {. } \\
\text { us. }\end{array}$ & $\begin{array}{cc}\text { Die } & \text { Au } \\
\text { des } & \text { Un }\end{array}$ & N. ischiadicus \\
\hline D. 1 & N. $p$ & Die Stre & N. tibialis. \\
\hline n. & & & \\
\hline $\begin{array}{l}\text { Die Einy } \\
\text { der des }\end{array}$ & $\boldsymbol{N}$. & $\mid \begin{array}{cc}\text { Die } & \mathrm{A} \\
\text { des } & \mathrm{Ft}\end{array}$ & $\boldsymbol{N}$ \\
\hline
\end{tabular}


Hiernach wird es erklärlich, wie an einem Gliede durch Unterbrechung der Leitungsfähigkeit in Nerven, welche die einer bestimmten Function vorstehenden Muskeln beherrschen, bei Integrität der die Antagonisten versorgenden Nerven die angeführten Deformitäten der Gelenke entstehen.

Leider läfst sich dieses anatomische Verhältnifs nicht von allen die Oberextremitäten beherrschenden in antagonistischer Beziehung zu einander stehenden Muskeln nachweisen. Viele in solcher Wechselbeziehung stehenden Muskeln der oberen Extremitäten erhalten ihre Nerven aus einem und demselben Aste. So verbreitet sich z. B. der N. radialis in dem M. triceps brachii und zugleich in einem seiner Antagonisten, den M. brachialis internus. Der Hauptantagonist des triceps jedoch, der $\boldsymbol{M}$. biceps, wird vom $\mathbb{N}$. cutaneus brachii medius, musculo-cutaneus und medianus versorgt. Dadurch ist immer noch genügend zu erklären, wie bei aufgehobener Function des Streckens und fortbestehender des Beugens und umgekehrt eine Deformität im Ellenbogengelenke entstehen kann. Dagegen erhalten z. B. der M. teres major und minor, obwohl jener den Arm nach innen, dieser ihn nach aufsen rollen hilft, gleichwohl ihre Nervenzweige aus dem $\mathbb{N}$, axillaris, während die anderen bei dieser Function mitwirkenden Muskeln der M. subscapularis einerseits und der No. supra- und infraspinatus andererseits vom Nervus axillaris und suprascapularis versorgt werden.

Unter solchen von der Physiologie bisher noch nicht ermittelten Umständen bleibt eben für jetzt Nichts übrig, als die Thatsache an sich zu constatiren, dafs in der Lähmung einzelnèr Muskelgruppen bei völliger Integrität ihrer Antagonisten die primäre Ursache vieler Deformitäten der Gelenke begründet ist.

Eine weitere Schwierigkeit bietet die Ermittelung des ursprünglichen pathischen Prozesses, von welchem die Lähmung einzelner Muskeln bedingt ist.

Heine (kurzer Bericht über die 25jährige Wirksamkeit der orthopädischen Heilanstalt in Canstalt 1854) und Rofs (zur Pathologie und Therapie der Paralysen, Braunschweig 1855) 
haben diese Frage einer Erörterung unterzogen. Ersterer leitet sie zum grölsten Theile von einer serösen Exsudation an den vorderen Spinalwurzeln her. Rols dagegen bringt gewichtige Gründe vor, dals diese Lähmungen von cerebralen Störungen abhangen. Bis jetzt sind uns aber die Endigungen der einzelnen motorischen Muskelnerven noch nicht bekannt und die pathologische Anatomie hat uns noch nicht genügende Auskunft über die diesen Lähmungszuständen zum Grunde liegende materielle Ursache im Nervensysteme zu geben vermocht. Wir sind daher auf das Gebiet der Hypothese angewiesen. Diese stïzt sich auf 2 Thatsachen: ist, und

1) dafs eine Lähmung einzelner Muskelgruppen vorhanden

2) dafs diese Lähmung heilbar ist.

Aus diesem letzteren Umstande ist zu folgern, dafs eine Degeneration der Nervensubstanz, sei es in der peripherischen Nervenbahn, sei es in der centralen, nicht vorhanden sein kann. Wohl aber kann ein Exsudat bestehen, welches die parlielle Lähmung zur Folge hat. Dieses Exsudat kann durch die Medication oder durch die Heilkraft der Natur zum Theil oder ganz resorbirt werden. In Folge dieses Heilungsprozesses kann entweder die Lähmung gänzlich oder bis zu einem gewissen Grade verschwinden. Ja es ist sogar wahrscheinlich, dafs selbst bei einer gänzlichen, aber vielleicht erst im Verlaufe vieler $\mathbf{J}$ ahre erfolgenden Resorption die gelähmten Muskeln durch die lange bestandene Unthätigkeit eine so bedeutende Gewebsveränderung erfahren haben, dafs sie trotz der wiederhergestellten Integrität der Nervenfunction in ihrer Unthätigkeit beharren und dadurch die Deformität unterhalten. Auch Rofs (I. c. S. 25.) theilt diese Ansicht, welche Romberg in seinem Lehrbuche über Nervenkrankheiten aufgestellt hat. (S. Ro mber g's Nervenkrankheilen, Motilitäts-Neurosen. Abthl. II. S. 147 u. 148.)

Es leuchtet ein, dals die Antagonisten der paralysirten Muskeln in ihrer gezwungenen Verkürzung so lange verharren müssen, bis sie entweder passiv gedehnt werden oder durch eine Wiederherstellung der paralysirten Muskeln zu ihrer normalen 
Thätigkeit gelangen. Je länger sie nun aber gezwungen sind, den verkürzten Zustand inne zu halten, desto mehr werden sie Veränderungen eingehen, sowohl in ihren contractilen und elastischen Verhältnissen, als in ihrem Gewebe selbst. Auch ihre Sehnen und Fascien müssen durch diesen andauernden Verkürzungszustand Veränderungen ihrer Struclur erfahren. Diese Veränderungen werden nun auch durch die pathologische Anatomie nachgewiesen. Die Structurveränderungen dieser verkürzten Muskeln gehen aber ungleich langsamer vor sich, als in den gedehnten paralysirten Antagonisten. Diese durch Obductionen nachgewiesene Thatsache rïhrt meiner Ansicht nach daher, dafs die Unthätigkeit dieser letzteren durch Unterbrechung der Nervenleitung bedingt ist, während die Innervation der verkürzten Muskeln ungestört besteht undihre Ausdehnung und normale Function nur indirect durch die Krankheit ihrer Antagonisten gestört ist.

Gleichwohl mufs auch diese durch irgend welche physiologische Eigenschaft des Muskels bedingte Verkürzung bei Jahre langer Dauer der Lähmung des Antagonisten zu so bedeutenden Veränderungen in dem Gewebe des verkürzten Muskels und seiner sehnigen Gebilde führen, dals die Verkürzung endlich der Ausdehnung widersteht, organisch wird. Aus der automatischen (physiologischen) Contraction des Muskels wird endlich eine pathologische Retraction desselben, d.h. eine nicht dehnbare, permanente Contractur. Diese permanent gewordene Contractur hat man nun aber oft irrthümlich für das stets wesentliche und primäre Moment der Deformität gehalten. Meine Beobachlungen haben mir die Ueberzeugung verschafft, dafs die Fälle, wo diese permanente Contractur das primäre ursächliche Moment der Deformität ist, bei Weitem die selteneren sind.

Blasius hat diesen Gegenstand in einer sehr interessanten Abhandlung einer ausführlichen Erörterung unterzogen (siehe Vierordt's Archiv. X. Jahrg. 2tes Heft: Ueber Stabilität der Theile und Stabilitäts-Neurosen). Blasius komml darin, wiewohl von eigenen neuen Gesichtspunkten ausgehend, ebenfalls 
zu dem Resultate, dem Nervensysteme den wesentlichsten Einflufs an den Deformitäten zu vindiciren. Es ist nicht thunlich, auf diese gehaltreiche Arbeit hier ausführlich einzugehen. Nur soviel möchte ich in der Kürze davon anführen, als zum Nachweise hinreichen dürfte: dafs die Ansicht dieses Autors und die meinige in den Ausgangspunkten zwar von einander abweichen, in den Endresultaten aber so sehr übereinstimmen, dafs daraus nothwendig dieselben therapeutischen Principien hervorgehen miissen.

Blasius bezeichnet mit "Stabilität" das Verharren der Theile in einer bestimmten normalen Lage durch den Einflus des Nervensystemes. Stabilitäts - Neurosen nennt er nun die Abänderung der Stabilität durch krankhafte Thätigkeit des Nervensystemes ohne Krampf, Lähmung oder organische Veränderung der betreffenden Muskeln. Als solche StabilitätsNeurosen stellt Blasius unter Anderen die dehnbare Contractur auf, in welcher er das Wesen vieler Rückgrats - und Gelenkverkrïmmungen erblickt. Blasius vermeidet so die Annahme der Paralyse als Ursache der Deformität und begnügt sich damit, die doch ein Mal vorhandene Unthätigkeit der Muskeln mit deren "Atonie" zu erklären. Jene, behauplet er, sei ein Unvermögen zur Action, diese ein permanent zu geringer Grad des Tonus, ein Fehler des ruhenden Zustandes. Dennoch räumt Blasius eine Verwandtschaft beider Zustände ein und gelangt zu dem Schlusse, dafs, wo eine Stabilitäts-Neurose sich als dehnbare Contractur gewisser Muskeln äufsert, in deren Antagonisten eine Atonie anerkannt werden müsse (s. l. c. S. 222 u. 223.). „Man müsse, erläutert Blasius dieses Verhältnifs ferner, bei der dehnbaren Contractur einer Muskelgruppe immer zugleich die Erschlaffung seiner Antagonisten im Auge haben und sie müsse als ein ebenso wichliges Moment wie die (dehnbare) Contractur betrachtet werden, so sehr, dals es wünschenswerth wäre, den Zustand gar nicht als Contractur zu benennen, sondern auf eine Weise, welche auf die Atonie und die Contractur zugleich hinweise." 
In den thatsächlichen Erscheinungen stimmt also die Darstellung von Blasius mit der meinigen überein. Blasius nämlich nimmt eine durch einen Nerveneinflufs bedingte dehnbare Contractur der verkürzten Muskeln einerseits und gleichzeitige Atonie der gedehnten Antagonisten andererseits an. Ich dagegen nehme einen durch primären Nerveneinflufs paralytischen Zustand der gedehnten Muskeln einerseits bei vollkommener Integrität der Antagonisten andererseits an, also die Paralyse, die nur ihrem Grade nach sehr verschieden sein kann, als das alleinige primäre ätiologische Moment der Deformität.

Abgesehen von den in den betheiligten Muskeln ermittelten pathologischen Befunden, von denen ich noch sprechen werde, beweisen therapeutische Facta die Richtigkeit meiner Ansicht. Eine hier anticipirte kurze Erwähnung dieser ist nicht ungerechtfertigt. Durch eine zweckmälsig gewählte therapeutische Einwirkung auf die von mir als paralytisch erkannten Muskeln, mittelst localer Galvanisation und localer Gymnastik, sind wir im Stande, die hier in Rede stehenden Deformitäten radical zu heilen. Eine directe Einwirkung dagegen auf die in dehnbarer Contraction befindlichen Muskeln, z. B. durch passive permanente Ausdehnung derselben mittelst mechanischer Apparate, kann im günstigsten Falle nur die Steigerung der secundären Zufälle verhüten, eine radicale Heilung der Deformität selbst aber niemals bewirken. Daraus ersieht man, wie auch hier die pathogenetische Auffassung nicht allein vom wissenschaftlichen Standpunkte aus, sondern auch vom praktisch-therapeutischen von höchster Wichtigkeit ist. Es ist kaum möglich, nach vorstehender Besprechung die hieher gehörigen Deformitäten mit anderen der, äufseren Form nach ähnlichen zu verwechseln. Daher bedarf die Begründung der differentiellen Diagnose nicht vieler Worte. Bei den Gelenkdeformitäten, also den Verkrümmungen des Oberschenkels und Kniegelenkes, dem Pes equinus, varus und valgus, dem Genu valgum, den entsprechenden an den Oberextremitäten, dem Caput obstipum, der Scoliosis und Kyphosis u. s. w. ist allemal eine im verschiedenen Grade von Relaxation bis zur Paralyse behinderte Thätigkeit der an der 
convexen Seite der Krümmung gelegenen Muskeln dann vorhanden, wenn momentan die deforme Stellung der Theile mit Leichtigkeit in die normale umgewandelt werden kann. Diese Ursache ist selbst dann noch anzunehmen, wenn, wie in sehr veralteten Fällen, in den verkürzten Muskeln, im knöchernen, knorpeligen und Bänderapparate der Deformilät schon so bedeutende Veränderungen eingetreten sind, dafs momentan die normale Stellung auch nur annähernd hergestellt werden kann. Mit wenigen Worten ausgedrückt, ist diese Art von Deformität mit Sicherheit überall anzunehmen, wo eine dehnb are Contractur der Muskeln vorhanden ist. Wo aber die verkürzten Muskeln nicht dehnbar sind, sind 3 Fälle möglich:

1) diese Muskeln waren im Anfange der Deformität dehnbar, haben aber durch die lange Dauer der Verkürzung eine organische Veränderung ihres Gewebes erlitten, wodurch nun permanente Contractur bedingt wird; oder

2) sie waren von Hause aus durch traumatische, rheumatische oder andere Krankheiten zur permanenten Contractur disponirt. In diesem Falle liegt die primäre Ursache direct in diesen verkürzten Muskeln; oder

3) die Ursache der Deformität liegt gar nicht in den Muskeln, sondern in den passiven Bewegungsorganen, Knochen, Knorpeln, Synovialhäuten, Sehnen, Aponeurosen und Fascien. Diese Fälle unterscheiden sich von den hier besprochenen durch die gänzliche Immobilität der betreffenden Gelenke und durch den Krankheitsverlauf.

Nach dieser pathogenetischen Auffassung haben sich mir bei der Behandlung zahlreicher Deformitäten folgende therapeutische Grundsätze praktisch bewährt:

Bei denjenigen Deformitäten, deren primäre Ursache in Paralyse der an der Convexität gelegenen Muskeln besteht, betrachte ich als die einzige rationelle Indication: Beseitigung der Paralyse. Um dieser zu genügen sind die locale Electrisation und die schwedische Heilgymnastik als die wesentlichsten und unentbehrlichsten Heilpotenzen von sachkundiger Hand anzuwenden. Diese beiden Mittel ergänzen sich 
auf unschätzbare Weise; jene zur Erweckung und Steigerung der Contractilität durch den elektrischen Strom, diese zur Hebung der Innervation, Nutrition und Energie in den paralysirten Muskeln durch locale, passiv oder mittelst des bewufsten Willensimpulses ausgeführte Bewegungen. Die Anwendung der localen Electrisation nach Duchenne geschieht mittelst eines Inductionsapparates täglich, je nach der Reizempfänglichkeit des Individuums während 10 bis 15 Minuten.

Die Anwendung der schwedischen Heilgymnastik geschieht in meinem Institute bei jedem mil einer musculären Deformität behafteten Kranken täglich 2 Stunden lang, innerhalb welcher unter Beobachtung der nöthigen Pausen die entsprechenden Bewegungen ausgeführt werden. Diese sind Anfangs mehr passiv. Mit der Zunahme der Contractilität gehe ich zu specifisch-activen Bewegungen der betreffenden Muskeln über. Die Heilung erfordert, wie es bei veralteten paralytischen Zuständen meistens der Fall ist, Geduld und Ausdauer von Seiten des Kranken und des Arztes. Mit diesen gelingt es, die gesunkene Innervation zu beleben. Mit der Beseitigung der Lähmung geht die der Atrophie der Muskeln Hand in Hand und mit der Herstellung des gestörten Muskelantagonismus erfolgt die Herstellung der normalen Form.

Neben der Electrisation und der schwedischen Heilgymnastik sind die anderweitigen, sonst wohl gerühmten Heilmittel auf diesem Gebiete von geringer Erheblichkeit. Stärkende Bäder haben für die Heilung von muskulären Deformitäten nur einen sehr bedingten Werth. Sie beziehen sich mehr auf die Kräftigung der allgemeinen Körperconstitution. Wo diese sie erfordert, mögen sie angewendet werden.

Die kalte Douche auf die relaxirten Muskeln applicirt, wird vielfach gerühmt und verdient es auch wohl, als Unterstützungsmittel mit herangezogen zu werden. Spirituöse Einreibungen auf die Gegend der paralysirten Muskeln verdanken ihren Nutzen mehr dem Acte des Reibens, als dem eingeriebenen Stoffe. Moxen und andere durch ableitende Secretionen wirkende Mittel passen während des die Paralyse bedingenden Exsudationspro- 
zesses, nicht aber in dem hier besprochenen Stadium, wo wir es nur mit den Residuen jenes Prozesses zu thun haben. Ich wende von allen diesen Dingen neben der Electrisation und der Gymnastik (oft auch nur neben letzterer allein) Nichts an. Dagegen bediene ich mich hier auch der mechanischen Apparate und glaube dadurch am thatsächlichsten zu beweisen, dafs ich, wo ich, wie bei Scoliose, den Nutzen mechanischer Apparate bestreite, nach wohlbegründeten Principien handle. Ich bin mir deshalb keiner Inconsequenz bewufst. So grofs auch die Analogie der Deformiläten der Extremitäten mit denen des Rumpfes ist, so bieten sich doch bei den letzteren für die Anwendung von Streck - und Druckapparaten leider erhebliche Contraindicationen dar. Diese bestehen in der Anwesenheit der wichtigsten und edelsten Organe in der Brust- und Bauchhöhle. Der schädliche Einflufs der Streck- und Druckapparate auf diese Organe ist es hauptsächlich, welcher, abgesehen von dem mehr als zweifelhaften Nutzen derselben bei den bezeichneten Rumpfdeformitäten, mich bestimmt, bei deren Behandlung der schwedischen Heilgymnastik abwechselnd mit mehrstündigem ruhigen Liegen während des Tages und Verhütung aller schädlichen Stellungen allein zu vertrauen. Ich habe Ursache mit den aus diesen Principien gewonnenen Heilresultaten vollkommen zufrieden zu sein.

Anders verhält es sich mit den Deformitäten an den Extremitäten. Die Beschaffenheit dieser gestattet die dem $Z_{w}$ ecke entsprechende Application mechanischer Hülfsmittel ohne Besorgnifs, edle, für das Gedeihen des Gesammtorganismus wichtige Organe in ihrer Function zu stören.

Gleichwohl mufs man auch hier in der Construction und Handhabung der anzuwendenden Apparate mit aller Umsicht und Sachkenntnifs verfahren. Der Arzt darf ihre Handhabung nicht den Kranken allein oder wohl gar dem Dienstpersonale überlassen, sondern mufs ihre Anwendung selbst überwachen. Die unrichtige Application schadet oft mehr, als die richtige nützen kann. Langwierige Excoriationen und vermehrle Renitenz der gesunden automatisch verkürzten Muskeln können als 
solche Nachtheile auftreten. Der Nutzen der mechanischen Apparate bei den in Rede stehenden durch Muskelparalyse bedingten Gelenkdeformitäten besteht darin, dafs das Gelenk möglichst permanent in der normalen Form erhalten und dadurch der Entwickelung der secundären Zufälle im knöchernen; knorpeligen und tendinösen Apparate vorgebeugt wird. Eine Radicalcur ist von denselben nicht zu erwarten, denn die Muskelparalyse besteht unter. der Anwendung auch der besten mechanischen Apparate fort, und ohne gleichzeitige Heilung der Paralyse muls die Deformität nach Ablegung des Apparates immer wieder in die Erscheinung treten. Innervation, Nutrition und Energie der Muskeln, die nothwendigen Requisite zur Radicalcur, werden durch Streckapparate nimmermehr bewirkt.

Eine solche Wirkung muls ich auch, und gewils ohne Vorurtheil, zur Herstellung des gestörten Muskelantagonismus den rein activen Bewegungen absprechen. Ich mag mich hier nicht auf den andererseits lebhaft angeregten Streit einlassen, ob zum diätetischen Gebrauche für Gesunde die schwedische sogenannte pädagogische Gymnastik oder die deutsche, das. sogenannte Turnen, besser sei. Allein die von dem Schweden Ling erfundene Heilgymnastik hat mit dem deutschen Turnen und den bei diesem angewandten activen Bewegungen gar nichts zu schaffen. Ihre Aufgabe besteht bei Behandlung dieser paralytischen Deformitäten darin, je nach der Localität die einzelnen Muskelgruppen, und zwar diese allein, entweder durch die passiven oder durch specifisch-active Bewegungen zur Contractilität zu bringen ( $\mathrm{s}$. meine Brochüren: Die schwedische Heilgymnastik. Berlin 1853. und: Mittheilungen aus dem Gebiete der schwedischen Heilgymnastik. Berlin 1854.). Beide Bewegungsformen können vom Kranken allein ohne Hülfe eines und oft mehrerer gymnastischer Assistenten nicht ausgeführt werden. Die vor Ling's Erfindung allein gekannten activen Bewegungen entsprechen nicht der hier zu lösenden Aufgabe. Denn es kommt hier allein darauf an, die gelähmten oder relaxirten Muskeln isolirt in Thätigkeit zu setzen, ohne gleichzeitige Mitbetheiligung der gesunden Antagonisten. Giebt man 
dem Kranken auf, vermöge seines Willensimpulses allein eine solche Einwirkung auf die die Deformitäten bedingenden kranken Muskeln zu üben, so vermag er dies eben nicht. Er wird vielmehr, gerade immer dem Heilzwecke entgegen, die verkürzten gesunden Muskeln, auf welche er selbstverständlich noch eine Einwirkung hat, zur Contraction bringen und so das die Deformität bedingende Mifsverhältnifs der Kraft dieser und der gelähmten Antagonisten vergrölsern. Eine solche nachtheilige active Gymnastik treibt eben schon jeder mit einer musculären Deformität behaftete Kranke durch den alltäglichen Gebrauch des deformen Gliedes. Darin liegt gewifs keine geringe Veranlassung zur unaufhaltsamen Steigerung der Deformität. Zur Heilung dieser können daher nur einzig und allein Bewegungen gebraucht werden, welche, wie die passiven und specifischactiven der schwedischen Heilgymnastik, auf die Erregung und Kräftigung der kranken Muskeln allein wirken.

Die Obductionsbefunde, welche in Betreff eines Vergleiches der Structurverhältnisse zwischen den verkürzten und verlängerten Muskeln angestellt sind, ergeben, wie ich bereits oben angedeutet, eine normale Farbe der verkürzten (gesunden) und eine grofse Blässe der verlängerten (gelähmten), (s. den Nachtrag zu dieser Abhandlung). So citirt z. B. Romberg einen, einen rechtseitigen paralytischen Klumpfufs betreffenden $\mathrm{Ob}$ ductionsbefund von Longet (s. Romberg: Nervenkrankheiten. Bd. II. Abthl. 2. S. 150.): „Sehr auffallend war die Blässe der gelähmten Muskeln, während die übrigen eine fast normale Farbe hatten. Die Entfärbung nahm die ganze Länge der Fasern in den Muskelbündeln ein. Die Nerven des rechten Beines waren dünner, als die der linken Extremität. Die vorderen Wurzelñ der den rechten Ischiadicus bildenden Lumbalund Sacralnerven hatten kaum ein Viertel des Durchmessers der entsprechenden linken Seite, während die hinteren Wurzeln auf beiden Seiten von normaler Dicke waren".

Ich führte oben bereits die fortbestehende Innervationsstörung als Grund davon an, dafs die verkürzten Muskeln, trotz ihrer gezwungenen Unthätigkeit, so lange den Structurverände- 
rungen widerstehen. Ein zweiter Grund dieser Erscheinung ist aber sicherlich die vom Kranken beim Gebrauche des Beines stets geübte Einwirkung auf die verkürzten Muskeln. Da diese sich aber trotz aller Bemühung des Kranken, so lange die Antagonisten gelähmt sind, nicht ausdehnen können, so wird alle Aclivität nur dazu dienen, die bereits vorhandene Verkürzung (Contractur) zu sleigern. Ganz anders verhält sich's mit den specifisch-activen Bewegungen der schwedischen Heilgymnastik. Es ist deren charakteristisches Wesen, eine Muskelgruppe allein in Thätigkeit zu setzen, bei gleichzeitiger Passivität der Antagonisten. Darin besteht ihr Vorzug und ihre ausschliefsliche Brauchbarkeit zur Heilung von allen denjenigen Deformitäten, welche durch Störung des Muskelantagonismus bedingt sind.

Für diejenigen, welche mit den specifisch-activen Bewegungen der schwedischen Heilgymnastik ganz unbekannt sind, mag ein Beispiel zur Erläuterung hier angeführt werden. Geselat, es habe Jemand in Folge von verminderler Thätigkeit der gemeinsamen Streckmuskeln des Unterschenkels eine Knieverkrümmung, welchen Zustand ich häufig an einem und beiden Schenkeln zu behandeln Gelegenheit hatte. Die Beugemuskeln sind gesund, müssen sich aber zusammenziehen, verkürzen, weil die Strecker mangelhaft fungiren. Dadurch ist die Kniegelenkverkrümmung bedingt. Die Heilung dieser Deformität hängt, so lange die verkürzten Beugemuskeln noch nicht organische Structurveränderungen eingegangen sind, einzig und allein von der Wiederherstellung der normalen Function der Streckmuskeln ab. Es koment daher darauf an, diese durch locale, d. h. auf sie allein beschränkte Gymnastik zu kräftigen, und zwar so, dals namentlich die gesunden Beugemuskeln dabei unthätig bleiben.

Zu diesem Zwecke giebt es nun vielfache Bewegungsformen, welche ich nach Professor Georgii "specifisch-active" nenne, weil sie die specifische Bethätigung eines Muskels oder einer Muskelgruppe bezwecken. Eine dieser Bewegungsformen für die gemeinsamen Strecker des Unterschenkels ist z. B. folgende: Das kranke Bein, nehmen wir an, es sei das linke, ruht mit der Mitte der hinteren Fläche des Oberschenkels auf 
einem gepolsterten Apparate, welcher elwa wie eine gewöhnliche Bank construirt, jedoch nur halb so breit und elwa $\frac{1}{2}$ Fuls höher ist. Das rechte Bein ruht mit der Planta pedis auf dem Boden. Vermag der Kranke aus Mangel eigener Kraft nicht diese Stellung inne zu halten, so wird er von Assistenten durch Fixirung des Rumpfes darin érhalten. Seine Hände stützt er zu beiden Seiten auf den bezeichneten Apparat. Der gymnastische Assistent, an der äufseren Seite des linken Beines stehend, legt seine linke Hand mit der Volarfläche auf die vordere Fläche des Unterschenkels dicht oberhalb des Fufsgelenkes, während er seine rechte dicht oberhalb des Kniegelenkes sanft auflegt. Er giebt nun dem Kranken auf, den Unterschenkel langsam zu strecken und macht, während der Kranke diese Bewegung ausführt, mit seiner am Unterschenkel anliegenden linken Hand einen dem Kraftmafse der kranken Streckmuskeln entsprechenden Widerstand. Nachdem dies geschehen, beobachtet man eine kleine Pause, dann drückt der gymnastische Assistent den mehr oder weniger gestreckten Unterschenkel langsam nieder, während der Patient denselben in gestreckter Stellung zu erhalten sucht, also seinerseits der Kraft des gymnastischen Gehülfen den ihm möglichen Widerstand entgegenselzt. Nach abermaliger Pause wird derselbe Act noch 2 oder $3 \mathrm{mal}$ wiederholt. Hat sich der Patient 5 Minuten lang ausgeruht, so geht man zu einer anderen, dem Heilzwecke entsprechenden passiven oder specifisch-activen Bewegung über.

Jeder, der diese Bewegung nach experimentiren will, wird sich überzeugen, dafs dabei der $\mathrm{Zweck}$, bei völliger Passivität der Beugemuskeln, die Streckmuskeln allein zu Contractionen zu bringen, vollständig erreicht wird. Es würde mich zu weit von dem vorgesteckten Thema entfernen, wollte ich specieller auf die Technik der Ling'schen Bewegungen eingehen, und verweise ich auf die darüber in meinen citirten Schriften gegebenen allgemeinen Angaben *).

*) Anmerk. Ich bin schon seit längerer Zeit mit der Ausarbeitung eines vollständigen Handbuches über die Technik der schwedischen Heilgymnastik und deren Erläuterung durch Abbildungen beschäftigt. 
Mit der nöthigen Geduld und Ausdauer ausgerüstet, bewirkt man nach und nach die Steigerung der Kraft und der Ernährung der so bethätigten Muskelgruppen und die Herstellung des gestörten Muskelantagonismus. Dagegen ist diese Behandlungsweise, sowohl die locale Gymnastik allein, als ihre Verbindung mit der localen Electrisation nicht mehr ausreichend bei der 2ten Gattung musculärer Gelenkverkrümmungen, bei welchen nicht mehr eine dehnbare, sondern eine organisch gewordene permanente Contractur, eine sogenannte Retraction vorhanden ist. Dies gilt sowohl für diejenigen Fälle, in welchen die Contractur anfangs dehnbar war, durch lange Dauer aber permanent wurde, als für jene, denen von Anfang an, also primär, eine permanente Contractur zum Grunde liegt.

Für diese Fälle beobachte ich im Allgemeinen folgende therapeutische Principien: Ich beseilige vor Allem die Muskelretraction je nach dem Grade und der Oertlichkeit durch gewaltsame Streckung unter Chloroformnarcose oder - durch die subculane Tenotomie. An diese schliefst sich im ersten Falle sofort, im zweiten nach Ablauf von 2 Tagen die entsprechende mechanisch-orthopädische Nachbehandlung an. Nach Verlauf von 6 Wochen beginne ich auch hier mit Anwendung der Heilgymnastik, wie ich es eben für diejenigen Fälle beschrieben, welche durch primäre Paralyse bedingt sind. Der Erfolg ist um so günstiger, wenn die Antagonisten der retrahirt gewesenen Muskeln nicht paralysirt sind. Aber auch in diesem Falle befinden sie sich je nach der Dauer der Deformität in Folge ihrer langen Unthätigkeit in einem so geschwächten $\mathrm{Zu}-$ stande, dafs ihre Kräftigung indicirt ist. Je schneller und vollkommener diese erzielt wird, desto sicherer ist der Erfolg der Cur.

Es bedarf nach der vorangegạngenen Darstellung kaum der Erwähnung, dafs ich mich nach erfolgter Vernarbung der durch die Tenotomie bewirkten Hautwunde derjenigen Apparate bediene, welche zur Erhaltung der normalen Stellung des Gliedes geeignet sind. Ebenso bediene ich mich, sobald die Kranken nach Operationen am Beine umherzugehen vermögen; 
entsprechender Apparate, um die durch die Beseitigung der Retraction gewonnene normale Form zu vervollständigen und dauernd zu erhalten. Hier haben die Apparate nicht nur einen präservativen Werth, sondern sie sind zur Erzielung eines günstigen Heilresultates unentbehrlich. Indem sie zur Innehaltung der möglichst normalen Stellung des betreffenden Gliedes dienen, befördern sie die Rückbildung der durch die lange vorhandene Muskelkrankheit secundär eingetretenen Verbildungen der betheiligten passiven Bewegungsorgane. So schätzbar in dieser Beziehung zweckmälsige Apparate sind, so bönnen sie doch die gleichzeitige heilgymnastische Nachbehandlung nicht entbehrlich machen. Durch diese wird die gesunkene Vitalität in den deformen Gliedern am sichersten wieder "gehoben. Jedermann weils, dafs diese deformen Glieder kalt, schlaff, atrophisch sind. Sie bedürfen einer erhöhten Innervalion und diese verschafft ihnen die locale Gymnastik, während beim Gebrauche der Apparate allein die Ernährung des Gliedes wenige und oft gar keine Fortschritte macht.

Ueber die Natur der bei den vorstehend besprochenen musculären Gelenkdeformiläten vorkommenden secundären Veränderungen in dem passiven Bewegungsapparate werde ich in einem Nachtrage meine Ansichten erörtern. Hier will ich nur noch Einiges über die Behandlung derjenigen Gelenkdeformitäten anschliefsen, welchen eine primäre Erkrankung des das Gelenk constituirenden passiven Bewegungsapparates zum Grunde liegt. Diese Deformitäten sind gröfstentheils die Endstadien pathischer Prozesse der Knochen, Knorpel, Ligamente, Fascien und Synovialmembranen. In einem oder dem anderen dieser genannten Gewebe beginnt ein oft unscheinender Krankheitszustand, welcher dieses und jenes Nachbargebilde in den Krankheitsprozels hineinzieht. In neuerer Zeit hat man sich nach Brodie's Vorgange bemüht, die differentielle Diagnose der Affectionen dieser verschiedenen Gelenksgewebe sicherer zu begründen, namentlich hat Soer mit der Bearbeitung des Brodie'schen Buches über Gelenkkrankheiten sich auf diesem Gebiete ein bedeutendes Verdienst erworben. Es ist dies nicht nur von 
wissenschaftlichem Interesse, sondern auch von prognostischem und therapeutischem Werthe, und dieser erstreckt sich selbst bis auf die Folgezustände dieser Gelenkkrankheiten, d. h. der Deformitäten. Und diese sind es, welche als mehr oder weniger feste anchylotische Gelenkverkrümmungen oft unter Fortdauer einzelner dem primären Krankheitsprozesse angehörigen Symptome Gegenstand der orthopädischen Behandlung werden.

Es ist nicht meine Absicht, hier den Nachweis dieses Nutzens zu liefern, den die differentielle Diagnose der Gelenkgewebeerkrankung auf die Behandlung hat. Es genüge nur die Andeutung, dafs diese exactere Anschauungsweise der Gelenkkrankheiten nach ihren Gewebstheilen einen bedeutenden Fortschritt auf diesem Gebiete in sich schliefst. Jedoch bedarf es noch sorgsamerer und zahlreicherer pathologisch-anatomischer Forschungen, um die bisherigen Beobachtungen zu berichtigen. So herrscht namentlich noch ein grofses Dunkel über die primär entzündliche Erkrankung der Knorpel, denen vor einiger Zeit noch alle Blutgefälse abgesprochen wurden, während Kölliker behauptet, bei einem 18jährigen Manne ganz deutlich Arterien im Gelenkknorpel der Epiphysen der langen Röhrenknochen gesehen zu haben. Ob dieselben auch im späteren Lebensalter vorkommen, darüber mangeln noch Beobachtungen. Das Gegentheil ist für jetzt die fast allgemein gültige Ansicht. Dennoch wird die Ulceration der Knorpel gerade vorzugsweise im millleren und höheren Lebensalter angetroffen. Unter solchen Umständen mulste man sich auf die Annahme beschränken, dafs die Knorpel nur secundär in Folge von Erkrankungen ihrer Nachbargebilde mit krankhaften Flüssigkeiten imbibirt und dadurch in ihrer Ernährung und Cohäsion verändert werden. So entbehrt ferner die Entzündung der Gelenkenden der Knochen noch so sehr der sicheren Kennzeichen, dals ihre genauere Unterscheidung von der entzündlichen Affection anderer Gebilde kaum möglich ist. Dagegen sind über die Entzündung der Synovialhäute durch die pathologische Anatomie und vergleichenden experimentellen Ergebnisse an Thieren die sichersten Aufschlüsse gewonnen. Es ist dies um so dankenswerther, als 
der Reichthum dieser Haut an Gefälsen und Nerven sie besonders häufig zu Erkrankung disponirt und ihre Ausbreitung dazu beiträgt, alle anderen Gebilde der Gelenke in den Kreis der Erkrankung hineinzuziehen.

So viele für die differentielle Diagnose mehr oder weniger brauchbare Symptome die Erkrankungen der einzelnen Gewebe auch darbieten, darin stimmen sie alle überein, dafs mit ihnen Functionsstörungen, Schmerz, Veränderung der Form und der Consistenz der Gelenktheile verbunden sind, und dafs sie häufig den Ausgang in Anchylose nehmen. Diese Anchylose wird dann vorzüglich wegen der durch sie bedingten gröfseren oder geringeren Functionsstörung Gegenstand der orthopädischen Behandlung.

Welche Fortschritte in neuerer Zeit die Therapie der Anchylosen gemacht hat, ist allgemein bekannt. Wir verdanken diesen Fortschritt vorzüglich der Erkenntnifs, dafs die Anchylose bei Weitem am häufigsten bedingt ist durch die abnorme, die $\mathrm{Be}$ wegung des Gelenkes hemmende Beschaffenheit der Weichtheile. Die frühere Ansicht, dafs die Anchylose meistentheils durch Verwachsung der knöchernen Theile des Gelenkes bedingt wäre, hat nur zu lange von therapeutischen Heilversuchen abgeschreckt, während die operative Chirurgie in neuerer Zeit selbst diese Fälle noch häufig mit dem glücklichsten Erfolge zu bekämpfen gelernt hat. Mittelst des Brisement forcé sind seit der Entdeckung des Chloroforms Kranke in den Besitz brauchbarer Gliedmalsen gelangt, deren kümmerliche Existenz kaum ein geringeres Unglück war, als der gänzliche Verlust. Wo das Brisement forcé unzulänglich blieb, werden noch durch die subcutane Osteotomie Resultate erzielt, von denen die ältere Chirurgie keine Ahnung hatle.

Ich mufs es mir versagen, dieses Gebiet mehr als andeutungsweise zu berühren. Dafs auch diese operativen Eingriffe vielfach gemifsbraucht worden sind, ist gewils. Ihre Anwendung war oft unnütz, nicht selten ganz schädlich und recht häufig überflüssig. Sie defswegen, wie z. B. Lorinser will (die Behandlung und Heilung der Contracturen im Knie- und Hüftge- 
lenke. Wien 1849.), zur Heilung der Anchylose ganz verwerten, ist trolz der günstigen Resultate, welche Lorinser durch die allmählige Streckung mittelst seines Streck- und Druckapparates erzielt hat, nicht zu rechtfertigen. A ehnliche Verfahrungsweisen durch solche die langsame Streckung bezweckende Apparate waren ohnehin schon längst im Gebrauche und namentlich leisteten in dieser Beziehung die Apparate von Delpech, Louvrier, besonders aber der von Bonnet (s. Traité de thérapeutique des maludies articulaires par A. Bonnet. Paris 1853. S. 318.) Alles, was man in dieser Beziehung erwarten darf. Unzählige Erfahrungen haben indefs dem kürzeren Verfahren ein zu wohlbegründetes Bürgerrecht erworben, als dafs es möglich wäre, es stets durch die langsame Streckung zu ersetzen. Die Mannigfaltigkeit der Fälle bietet vielmehr genïgende Gelegenheit fïr beide Heilmethoden dar.

Beiläufig gestalte ich mir noch eines Verfahrens zu erwähnen, welches sich mir bei einer häufig vorkommenden Gelenkkrankheit, dem aus chronischer Entzündung der Synovialhaut entstehenden Hydrarthron und einigen anderen Arten von falscher Anchylose, in hohem Grade nützlich erwiesen hat. Ich meine die Anwendung passiver Bewegungen nach Bonnet. Zu meiner nicht geringen Ve:wunderung habe ich in der deutschen medicinischen Litteratur bisher eine Mittheilung darüber nicht gefunden, dafs von diesem Verfahren in Deutschland Gebrauch gemacht worden sei. Jeder Arzt, der einige Erfahrung in der Behandlung derartiger Gelenkkrankheiten hat, weils, wie hartnäckig dieselben oft allen äufseren und inneren Curmethoden widerstehen. Ich habe das Hydrarthron z. B. Jahre lang unter der Anwendung von heroischen Mitteln, dem Cauterium actuale und potentiale, den Bepinselungen mil der stärksten Auflösung von Argentum nitricum, mit der Tinct. jodi und darauf folgender Application einer sehr starken Sublimatsolution, dem innerlichen Gebrauche des Sublimats, des Jodkali u.s.w. trotzen sehen. Selbst wenn die Absonderung der Synovia sich vermindert hatte, wenn ich durch gewaltsame Streckung die vom Hydrarthron abhängige. Winkelstellung des 
Gelenkes, namentlich am Knie, beseitigt hatte, blieb oft eine störende Empfindlichkeit im Gelenke und eine durch gehinderte Beweglichkeit desselben bedingte Unbrauchbarkeit des Gliedes zurück.

Diese Symptome weichen der consequenten Anwendung der passiven Bewegungen. Zur Ausführung dieser hat Bonnet sehr sinnreiche Apparate für die verschiedenen Gelenke construiren lassen, mittelst welcher der Kranke selbst diese in Betreff des kranken Gelenkes völlig passiven Bewegungen ausführt. In dem citirten Werke befinden sich höchst anschauliche Abbildungen dieser Apparate, nach welchen sie jeder Mechaniker anfertigen kann.

Bekanntlich war es bisher allgemein gültige Regel, schmerzhafte chronische Gelenkleiden durch Ruhe zu behandeln. Man ward darin durch die stete Beobachtung bestärkt, dafs Bewegùng den Schmerz und die Unbrauchbarkeit der kranken Extremität steigert. Auch Brodie besteht auf dieser Ansicht; allein es ist nicht zu leugnen, dafs die Besserung bei aller noch so lange ausgedehnten Ruhe sich oft vergebens erwarten lälst. Man versuche es mit den passiven Bewegungen!

Ist das Leiden z. B. am Knie, so kann der Kranke dabei die horizontale Lage inne halten. Der dazu erforderliche Apparat ist so construirt, dafs der Oberschenkel mittelst Hohlschiene und Riemen fixirt ist. Diese Schiene steht in leicht beweglicher Verbindung mit einer zweiten Hohlschiene zur befestigten Aufnahme des Unterschenkels. Diese untere Schiene wird durch die Hand des Kranken selbst miltelst einer über eine Rolle gehenden Schnur in auf- und abwärts steigende Bewegung gesetzt. Man könnte diese passive Streckung und Beugung auch ohne Apparat durch die Hände eines Gehülfen verrichten lassen. Allein diese Bewegungen müssen mehrmals täglich $\frac{1}{4}$ bis $\frac{1}{2}$ Stunde lang angewendet werden, und dies wäre eine ungeheuer anstrengende Arbeit für die Gehülfen. Davon aber auch abgesehen, mufs dabei jede gewaltsame Erschülterung vermieden werden, und dies ist durch den Apparat, den der Kranke selbst in Bewegung setzt, an sichersten zu er. 
reichen. Ich kann Bonnet's Ausspruch lediglich bestätigen, dafs die von ihm angegebenen Apparate dem Manöver eine Leichtigkeit, Zartheit und Gleichmälsigkeit gewähren, welche man bei jedem anderen Vorgehen vergebens suchen würde.

Anfangs verursachen diese Bewegungen eine geringe $\mathrm{Zu}-$ nahme des Schmerzes, allein mil jedem Tage nimmt dieser ab, die Geschwulst vermindert sich, die Articulation wird freier. Ist mit dem chronisch-entzündlichen Gelenkleiden eine Contractur verbunden, so vollzieht man die Streckung auf schnellem oder langsamem Wege und schreitet etwa nach Verlauf von 8 Tagen zur Anwendung dieser künstlichen passiven Bewegungen. Bonnet hat die Anrvendung dieser Bewegungen selbst auf bedeutende destructive Gelenkleiden ausgedehnt. Nach meinen Erfahrungen kann ich seinen Ausspruch bestätigen, dals mittelst derselben mehrere bishẹr unlösbare Probleme gelöst werden. Ob sie aber, wie Bonnet (l. c. S. 19.), selbst ulcerirten Gelenkknorpeln sowohl ihre Form als Glätte wiedergeben, möchte schwer zu beweisen sein. Jedenfalls sprechen sowohl meine Erfahrungen, als die mehrerer französischer Aerzte für die hohe Wirksamkeit dieser Bewegungen in gedachten Fällen. Teissier, nach B onnet's Angabe, ein ausgezeichneter Practiker zu Lyon, will die Ruhe beim Tumor albus ganz vermieden wissen. Auch ich habe bei diesem Gelenkleiden die Kranken den ganzen Tag umher gehen lassen, jedoch mit der Vorsicht, dafs das kranke Gelenk mittelst eines unbeweglichen Stützapparates vor jeder activen Bewegung geschützt bleibt, wodurch namentlich jeder Druck auf die Gelenkfläche vermieden wird. Welchen Nutzen dieses Umhergehen für die ganze Körperconstitution, im Vergleiche zu dem andauernden Liegen hat, bedarf kaum der Erwähnung. Allein man bedarf dazu vollkommen zweckmälsig construirter Apparate, wenn nicht Entzïndung und Deformität dadurch vermehrt werden sollen.

Citire ich noch die Ansicht Mellet's (s. dessen Manuel pratique d'orthopédie. p. 336.), so geschieht es in der Absicht, einem Heilverfahren Nachahmung zu verschaffen, welches bei gröfster Einfachheil geeignet ist, eine schwer empfundene Lücke 
im Gebiete chronischer Gelenkkrankheiten in willkommener Weise auszufuillen. Dieser Autor äufsert sich darüber folgendermalsen: „Die Chirurgen empfehlen im Allgemeinen die vollkommenste Ruhe und scheuen jede Bewegung, um die Entzündung nicht zu vermehren. Indefs unterscheidet sich die den weifsen Geschwülsten zum Grunde liegende besondere Art von Entzündung nach meinen Erfahrungen von der Natur anderer Entziindungen. Mehrere Beobachtungen haben mich im Gegentheile veranlafst, jeden Tag an den Gelenken, welche den Sitz der Krankheit bilden, leichte Beuge - und Streckbewegungen ausführen zu Iassen, welche nach und nach zu steigern sind. Weit entfernt, den Schmerz zu vermehren und das Uebel zu verschlimmern, hat mich die Erfahrung an 6 hierher gehörigen, seit einigen Jahren geheilten Fällen gelehrt,- dafs man mit diesem einzigen Mittel weiter kommt, als mit der Ruhe und allen gewöhnlich in Gebrauch gezogenen Heilapparaten. In der That sah ich unter der Einwirkung dieser Bewegungen die Kniegeschwulst fallen, die fistulösen Abscesse, welche sich bei Einigen gebildet hatten, sich entleeren und bald vernarben, den Anfangs aufserordentlich heftigen Schmerz nach und nach weichen, so dals ich mir nicht nur sehr ausgedehnte Bewegungen, sondern selbst die Application eines mechanischen Apparates gestatten durfte, mittelst dessen ich die mit den Händen bewirkte Extension unterhalten konnte. Ich betrachte daher diese Bewegungen als äufserst nützlich für die Behandlung der weilsen Gelenkgeschwülste, sei es, um darin eine freie Erregung herbeizuführen, in welcher die krankhafte untergeht, sei es, um die Beugung des Gelenkes zu verhülen und zu heilen."

Fern von jeder Einseitigkeit und in gerechter Würdigung aller irgend wie nützlicher äufserer und innerer, diätetischer und medicinischer Heilpotenzen, legt Bonnet mit Recht zur Bekämpfung chronischer Gelenkkrankheiten einen grofsen Werth auf diese künstlichen passiven Bewegungen. Ich kann ihm nur aus voller Ueberzeugung zustimmen, wenn er hohen Werth auf eine solche functionelle Therapie der Gelenkkrankheiten legt, und wenn er sie als solche bezeichnet, die dazu 
bestimmt ist, neben der operativen Chirurgie und der Matevia medica eine wichtige ergänzende Stelle einzunehmen. $\mathrm{Es}$ ist zu bedauern, dals Bonnet mit den practischen Ergebnissen der schwedischen Heilgymnaslik unbekannt ist. Um so überraschender sind seine Bemerkungen über den Nutzen der speciellen Bewegungen. So sagt er in der Vorrede des citirten Werkes S. XVII.: „die Zeit ist nicht fern, wo ich mitlelst Bewegungen des Thorax die von Affectionen der Rippenarticulationen abhängigen Oppressionen mit gleichem Erfolge bekämpft haben werde, wie ich es bei den Dyspnöen gethan habe, welche von bedeutenden Thoraxdeformitäten abhangen. Einige Thatsachen gestatten mir selbst die Hoffnung, dafs man mit nicht geringerem Erfolge auf die Neuralgien und Lähmungen einzuwirken vermag, deren Ausgangspunkt das von einem gesunden Rückgrat umgebene Rückenmark ist, als bei dessen in Folge von Gibbositäten eintretenden Erweichungsprozessen".

Ich ersehne mit ihm die Zeit herbei, wo die Therapie neben der Medication behufs Bekämpfung der Ursachen und der vitalen Umänderung verschiedener Krankheitszustände auch der speciellen physiologisehen Function der Organe in ausgedehnterer Weise Rechnung tragen werde. Die physiologische Function der Gelenke ist die Bewegung, und in der Regulirung der. Bewegung besitzen wir ein Heilmittel für mannigfache chronische Gelenkkrankheiten, das nach meinen Erfahrungen den von Bonnet angeregten Erwartungen entspricht und allgemeine Anwendung verdient. 